\title{
Reviewing the consequences of genetic purging on the success of rescue programs
}

\author{
Noelia Pérez-Pereira $^{1}\left[\right.$ Armando Caballero $^{1}\left[\right.$ A $^{-} \cdot$ Aurora García-Dorado $^{2}(\mathbb{C}$
}

Received: 5 June 2020 / Accepted: 18 July 2021 / Published online: 19 October 2021

(c) The Author(s) 2021, corrected publication 2021

\begin{abstract}
Genetic rescue is increasingly considered a promising and underused conservation strategy to reduce inbreeding depression and restore genetic diversity in endangered populations, but the empirical evidence supporting its application is limited to a few generations. Here we discuss on the light of theory the role of inbreeding depression arising from partially recessive deleterious mutations and of genetic purging as main determinants of the medium to long-term success of rescue programs. This role depends on two main predictions: (1) The inbreeding load hidden in populations with a long stable demography increases with the effective population size; and (2) After a population shrinks, purging tends to remove its (partially) recessive deleterious alleles, a process that is slower but more efficient for large populations than for small ones. We also carry out computer simulations to investigate the impact of genetic purging on the medium to long term success of genetic rescue programs. For some scenarios, it is found that hybrid vigor followed by purging will lead to sustained successful rescue. However, there may be specific situations where the recipient population is so small that it cannot purge the inbreeding load introduced by migrants, which would lead to increased fitness inbreeding depression and extinction risk in the medium to long term. In such cases, the risk is expected to be higher if migrants came from a large non-purged population with high inbreeding load, particularly after the accumulation of the stochastic effects ascribed to repeated occasional migration events. Therefore, under the specific deleterious recessive mutation model considered, we conclude that additional caution should be taken in rescue programs. Unless the endangered population harbors some distinctive genetic singularity whose conservation is a main concern, restoration by continuous stable gene flow should be considered, whenever feasible, as it reduces the extinction risk compared to repeated occasional migration and can also allow recolonization events.
\end{abstract}

Keywords Migration $\cdot$ Gene flow $\cdot$ Reconnection $\cdot$ Inbreeding depression $\cdot$ Population extinction

\section{Introduction}

Genetic rescue is the reduction of the extinction probability of endangered populations through the introduction of migrant individuals. Genetic rescue programs have been successful in multiple occasions (Vilà et al. 2003; Fredrickson et al. 2007; Johnson et al. 2010; Åkesson et al. 2016; Weeks et al. 2017; Hasselgren et al. 2018; Ralls et al. 2020), and are considered a promising strategy in Conservation Biology

Aurora García-Dorado

augardo@ucm.es

1 Centro de Investigación Mariña, Universidade de Vigo, Facultade de Bioloxía, 36310 Vigo, Spain

2 Departamento de Genética, Fisiología y Microbiología, Facultad de Biología, Universidad Complutense, 28040 Madrid, Spain
(Waller 2015; Tallmon 2017). However, most information on their consequences refer to a few generations (usually one or two, rarely six, Whiteley et al. 2015). Furthermore, concern has been raised by the extinction of the Isle Royale wolves population, where the genetic contribution of a single migrant wolf from the large mainland population quickly spread in the resident population thanks to the breeding vigor of its offspring, possibly causing an increase in inbreeding and an associated fitness decline that triggered population extirpation (Hedrick et al. 2014, 2017, 2019). Therefore, despite the multiple studies supporting the practice of genetic rescue (Frankham 2015; Kolodny et al. 2019), its consequences in the medium to long term remain uncertain (Hedrick and Fredrickson 2010; Hedrick and GarcíaDorado 2016; Bell et al. 2019; Kyriazis et al. 2020). Here we review the main theoretical aspects behind the impact of inbreeding depression and purging on the long-term success 
of rescue programs and carry out computer simulations to evaluate the predicted outcome of these programs under some specific scenarios.

\section{Some background on purging and on its role during genetic rescue}

From the genetic point of view, the main determinant of early future extinction of small endangered populations is the inbreeding depression of fitness (O'Grady et al. 2006; Allendorf et al. 2013; Frankham et al. 2014). This is due to the expression, as inbreeding accumulates, of the initial inbreeding load $B$, often interpreted in terms of lethal equivalents (Morton et al., 1956). Here we deal with the inbreeding load $B$ ascribed to the recessive deleterious component of many rare detrimental alleles that remains hidden in the heterozygous condition in a non-inbred population (see, e.g., Caballero 2020, Chap. 8), and we do not consider the possible inbreeding load ascribed to overdominance. According to theory, in stable populations the inbreeding load is expected to be larger for populations with larger effective size $N$ (see Eq. 13 in García-Dorado 2007), the increase being much more dramatic for more recessive deleterious alleles (García-Dorado 2003; Hedrick and García-Dorado 2016). Thus, a historically large population can be genetically healthy in the sense of showing a high average fitness but, still, its individuals are expected to be heterozygous for many rare (partially) recessive deleterious alleles.

Due to the reduction of $N$ in an endangered population, both drift (the dispersion of gene frequencies due to random sampling of alleles) and inbreeding (the increase in homozygosis in the offspring of related individuals) increase through generations. Inbreeding increases the expression of recessive deleterious effects in homozygosis, which produces inbreeding depression but also triggers an increase of selection against the deleterious alleles known as genetic purging. The process is described by the Inbreeding-Purging model (IP model in García-Dorado 2012), according to which the fitness expected by generation $t\left(W_{t}\right)$ after a reduction of $N$ is predicted as in the classical Morton's et al. (1956) model, but replacing Wright's inbreeding coefficient $F$ with a purged inbreeding coefficient $g$ that is weighed by the ratio $q_{t} / q_{0}$, where $q_{0}$ is the frequency of the deleterious allele in the original non-inbred population and $q_{t}$ is the corresponding value expected from purging by generation $t$ :

$W_{t}=W_{0} \exp \left(-B g_{t}\right)$,

where $W_{0}$ and $B$ are, respectively, the expected fitness and the inbreeding load in the initial non-inbred population, and where $g_{t}$ can be predicted as a function of the effective population size $N$ and of the recessive component of the deleterious effects, i.e., the purging coefficient $d$ which, for a given homozygous effect $s$ and dominance coefficient $h$, amounts $d=s(1-2 h) / 2$. Thus, $B$ is the sum of $2 d\left(1-q_{0}\right) q_{0}$ over all the sites with segregating deleterious alleles.

Similarly, the corresponding inbreeding load at generation $t\left(B_{t}\right)$ can be predicted as

$B_{t}=B g_{t}\left(1-F_{t}\right) / F_{t}$,

which accounts for the joint reduction of the inbreeding load ascribed to drift and purging, that is faster than under drift alone.

The efficiency of purging can be defined as the proportional reduction of the original deleterious allele frequencies that it is expected to cause, i.e., the expected $\left(q_{0}-q_{t}\right) / q_{0}$. Therefore, since the asymptotic value of $g$,

$\hat{g}=\frac{1-2 d}{1+2 d(2 N-1)}$

predicts the asymptotic value of $q_{t} / q_{0}$ to a good approximation for $N d \geq 1$, we predict the efficiency of purging as $(1-\hat{g})$. This expression accounts for the opposing effects of purging and genetic drift after long-term inbreeding, when all the deleterious alleles responsible for the initial $B$ are expected to be fixed or lost. It shows that the efficiency of purging increases with increasing $N d$ being, for any given $d$ value, higher in large populations (i.e., under slower inbreeding) than in small ones. As $d$ approaches 0 , $\hat{g}$ approaches 1, and the role of purging preventing deleterious fixation becomes negligible compared to that of drift. As $N d$ increases, $\hat{g}$ goes to zero, drift becomes irrelevant and the deleterious alleles responsible for $B$ in the original non-inbred population are expected to be virtually removed by purging. Figure 1 illustrates that purging occurs faster under faster inbreeding (i.e., for smaller $N$ ) but it is also less efficient.

In agreement with the above predictions, there is evidence that purging is able to reduce an important fraction of the inbreeding depression in populations with effective sizes about ten or above, while faster inbreeding (as continued full-sib mating or effective sizes below 10) seems to promote purging just against lethal or severely deleterious mutations (Templeton and Read 1984; Hedrick 1994; Wang et al. 1999; Ávila et al. 2010; Pekkala et al. 2012; Bersabé and GarcíaDorado 2013; López-Cortegano et al. 2016; Caballero et al. 2017).

Therefore, the success of genetic rescue programs to reduce the extinction risk ascribed to inbreeding depression depends on the balance between two different effects of gene flow. On the one hand, migrants reduce inbreeding, thus causing an increase of fitness that corresponds to reversed inbreeding depression, which is known as hybrid vigor or heterosis (Falconer and Mackay 1996, p. 253; Caballero 2020, p. 196) and is due to the introduction of 


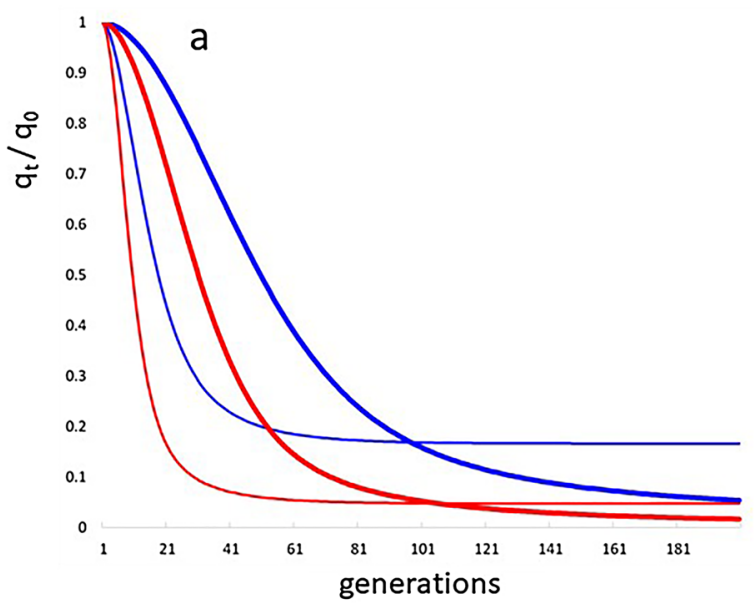

Fig. 1 Consequences of purging over 200 generations after the effective population size of an ancestral large population with $W_{0}=1$ and $B=2$ drops to $N=10$ (thin lines) or $N=100$ (thick lines); blue: $s=0.2, h=0, d=0.1$; red: $s=0.5, h=0, d=0.25$; black: neutral (no purging) predictions. a Average of the frequency of the deleterious alleles of the ancestral population through generations relative to the corresponding initial frequency $\left(q_{t} / q_{0}\right.$, inferred as $\left.g_{t} / F_{t}\right)$. In the absence of selection this average relative frequency would remain equal to 1 . However, it is substantially reduced due to purging. The reduction occurs faster for smaller $N$. After some time, an equilibrium

the beneficial allele at some of the sites where the individuals of the endangered populations were homozygous for the deleterious allele. Obviously, the more purging occurred before migration, the smaller is the inbreeding depression accumulated in the endangered population and the corresponding hybrid vigor induced by the rescue program. On the other hand, migrants bear their own inbreeding load due to partially recessive deleterious alleles hidden in heterozygosis. This hidden inbreeding load may fuel future inbreeding depression in the endangered population, which can be mitigated by purging. Therefore, the success of genetic rescue programs can critically depend on the purging occurring on both the donor population and the endangered recipient one. The impact of a rescue program on the extinction risk also depends on many other factors besides inbreeding and purging, such as the possible advantage due to migrants contributing new adaptive mutations accumulated in the donor population after the isolation of the endangered one, the possible adaptive disruption if the two populations are adapted to different environments, the increased resilience due to restoration of adaptive potential, the introduction of demographic and environmental stochasticity or other factors related to management as the risk of spread of infectious diseases, etc. (Ralls et al. 2020). However, in this review we will focus on genetic purging considering theoretical predictions and available evidence, including new simulation results, to understand its role as a determinant of the success is reached where the average relative frequency represents the fraction of ancestral deleterious alleles that become fixed because they have not been purged. This asymptotic average frequency is larger for smaller populations, indicating less efficient purging. Purging is quicker and more efficient for larger $d$ values; $\mathbf{b}$ Expected average fitness through generations showing initial inbreeding depression and later substantial recovery due to purging, although never up to its ancestral value. A more comprehensive model including non-purging selection and new mutation (the Full Model) can also be found in García-Dorado (2012)

of genetic rescue programs in reducing both inbreeding depression and extinction risk through generations.

\section{Genetic purging in the donor population}

Drawing migrant individuals from large, genetically healthy populations has usually prompted population recovery during a few generations (Whiteley et al. 2015; Ralls et al. 2020). Nevertheless, as explained in the previous section, migrant individuals sampled from a historically large donor population are expected to be heterozygous for many rare (partially) recessive deleterious alleles. Each of these alleles cause slight or no damage on the fitness of migrants and of the offspring they produce when mating individuals of the recipient population. However, they may contribute inbreeding load to the recipient population that can cause an increase of the inbreeding depression in the future. Thus, using large donor populations to rescue very small endangered ones could in theory enhance the risk of extinction from future inbreeding depression.

Therefore, in some cases, migrants from slowly inbred efficiently purged populations (i.e. where inbreeding has accumulated due to effective population sizes above several tens), could be a better alternative in the medium to long term. These migrants can produce hybrid vigor without a substantial increase of the inbreeding load and of the long-term extinction risk, even if leading to smaller gains in genetic diversity and, therefore, in adaptive potential. It has 
been stated that reliable evidence is required about the superiority of migrants sampled from small populations (Ralls et al. 2020) but, in fact, except when considering just a few generations, reliable evidence is required for the success of rescue using both small and large donor populations.

It needs to be remembered that purging becomes less efficient for smaller populations. Therefore, using migrants from a population that underwent drastic bottlenecking can introduce high genetic load as well as little genetic diversity and adaptive potential, bringing together the worst of both worlds. This seems to have been the case with one of the donor populations used to rescue the endangered Pacific pocket mouse (Wilder et al. 2020). Fortunately, analysis of genomic data can provide inferences on the demographic history (Santiago et al. 2020, and references therein) that may allow the election of a donor population with a record of moderate size allowing for efficient purging.

It has been proposed that the increase of extinction risk ascribed to the deleterious alleles introduced during genetic rescue can be controlled by prioritizing a lower putative load inferred from genomic analysis over a high genetic diversity (Kyriazis et al. 2020; Teixeira and Huber 2021). However, relying on the ability to identify the mutations that are responsible for a main fraction of the fitness load is not free of perils (Kardos and Shafer 2018; Ralls et al. 2020; García-Dorado and Caballero 2021). For example, the number of putatively deleterious variants per genome has not been found to be a reliable predictor of inbreeding depression in island populations of foxes and wolves (Robinson et al. 2018). Similarly, the Homozygous Mutation Load, defined by Keller et al. (2011) as the number of homozygous loci for rare alleles carried by an individual, used as a proxy of the fitness load due to homozygous (partially) recessive deleterious mutations, has only a moderate expected correlation with phenotypic values of individuals in large populations (Caballero et al. 2020). The recent evidences that purging can reduce different genomic proxies for the fitness genetic load (Xue et al. 2015; Robinson et al. 2018; Van der Valk et al. 2019; Grossen et al. 2020) suggest that genomic analyses could be helpful to infer the inbreeding load at the population level, but this is more likely to be useful in identifying suitable donor populations than optimal migrant individuals. However, even identifying the best donor population is not straightforward based on genomic information, as between population differences in fitness inbreeding load can be mainly due to a very small fraction of the annotated alleles of any deleterious category. For example, considering two populations with a common origin but different demographic history, one of them could have purged many more mildly deleterious alleles than the other during a long evolutionary period, but the other one could have purged a few more severely deleterious alleles during a recent shorter period with a relatively smaller size. Thus, the population with the smallest count of putatively deleterious alleles per genome is not necessarily the donor population introducing the smallest fitness inbreeding load.

Thus, when the donor's inbreeding load is a concern (see next section), it can be safer preferring donor populations that have gone through a period of moderate effective size allowing for purging in the past than choosing migrant individuals on the basis of their low burden of putatively deleterious alleles. Adaptive potential could be further improved by using different donor populations if available. For example, some of the populations of Canadian lynx in eastern North America could need to be rescued in the future due to global warming preventing natural migration through natural ice bridges. Then, migrants could be sampled from the several peripheral populations in eastern Canada that are under continuous partial isolation instead of from the large mainland Canada population (Koen et al. 2015).

A relevant question is in which situations the load introduced by non-purged migrants can be more harmful than the inbreeding depression they remove. The answer depends on the purging processes that take place in the recipient endangered population, analyzed in the next section.

\section{Purging occurring in the recipient population}

\section{Theoretical arguments}

Let us now think of a rescue program where the donor population has a long history of large effective population size, so that it can be considered genetically healthy (i.e. it shows little reduction of mean fitness from segregating and fixed deleterious alleles) but it is non purged (i.e., it hides large inbreeding load). The success of the rescue program depends on the balance between the inbreeding depression of the endangered population that is intended to be reversed by the migrant gene flow and on the future depression that can arise from the load concealed in the migrant individuals.

In the past, when the endangered population began to shrink, the inbreeding load $B$ of the ancestral non-endangered population fueled both inbreeding depression and purging. As predicted by Eqs. (1) and (2), the slower was the increase of inbreeding during that process (i.e., the larger was $N$ ) the slower but more efficient was purging (Eq. 3 and Fig. 1). This means that, the slower was the process that led the recipient population to its current inbreeding level: a) the smaller is its expected inbreeding depression, so that less hybrid vigor is expected after migration; $b$ ) the smaller inbreeding load it hides, so that migrant individuals coming from a large population are more likely to carry more (partially) recessive deleterious alleles than resident ones. In addition, if the recipient population is very small by the time of migration and does not experience a quick demographic recovery, the load introduced by migrant individuals will 
not be efficiently purged. The result is that, if the recipient population has a history of slow inbreeding previous to migration but remains very small after that, migration events could in some cases reduce fitness in the medium to long term. On the contrary, migration events are expected to be particularly beneficial for populations with a history of drastic bottlenecking that have recovered a moderate size allowing future purging.

\section{A simulation illustration}

To assess the relevance of the purging occurring in the endangered recipient population we performed a simulation analysis that is summarized in the Boxes below and is reported with more detail in the Supplementary Material.

\section{Box 1: Purging and fitness rescue}

First, we simulated a large non-threatened population of $N=10^{4}$ individuals for $10^{4}$ generations to approach the mutation-selection-drift equilibrium. Then, smaller threatened populations were derived and different scenarios were simulated as shown in Fig. 2. In a first phase, threatened populations with different sizes $\left(N_{1}=4,10\right.$ or 50$)$ were maintained for $t=N_{1}$ generations (e.g., up to generation 50 for populations with $N_{1}=50$, etc.), so that the average inbreeding coefficient was $F \approx 0.4$. Then, in a second phase with population size $N_{2}$, each threatened population was maintained with the same constant size $\left(N_{2}=N_{1}\right)$, or with a different size, and entered or not a genetic rescue program. Each two-phase scenario is denoted by the corresponding population sizes (e.g., $50-10$ for $N_{1}=50$ and $N_{2}=10$ ). Rescue consisted of the addition of males randomly sampled from the large $N=10^{4}$ population. Between 500 and 2500 replicated rounds were simulated. The number of individuals introduced during each migration event was five for lines with $N_{2}=50$ and one for lines with $N_{2}=10$ or $N_{2}=4$. Regarding the number of migration events, we considered four strategies: (i) a single event; (ii) two events with an interval of five generations; (iii) periodic migration every five generations; and (iv) the "one migrant per generation" (OMPG) strategy, that is widely recommended to retain connectivity in metapopulation management (Mills and Allendorf 1996). All the sizes considered for the threatened populations (4, 10 and 50) correspond to the IUCN Red List category of Critically Endangered or Endangered according to Criterion D (IUCN 2012).

Non-recurrent deleterious mutations occurred at rate $\lambda=0.2$ per gamete and generation in free recombining sites, with fitness $1,1-s h, 1-s$ for the wild-type homozygote, the heterozygote and the homozygote for the mutant allele, respectively. The inbreeding load $B$ was calculated as the sum of $s(1-2 h) p q$ for all selective sites, where $q$ and $p=1-q$ are the frequencies of the mutant and wild allele, respectively (Morton et al. 1956). The homozygous

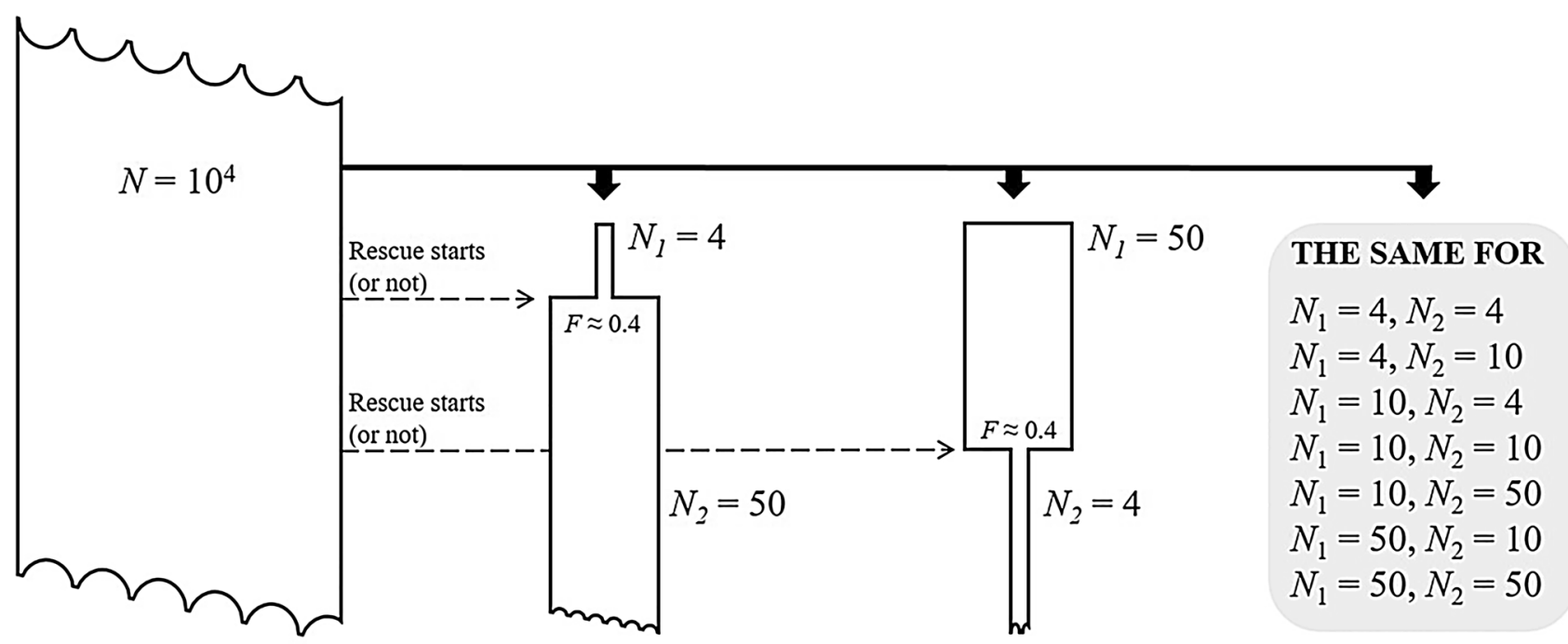

Rescue: once*, twice*, periodic* or OMPG

* one migrant per event if $N_{2}=4$ or 10; five migrants if $N_{2}=50$

Fig. 2 Simulation scheme. A small number of individuals $\left(N_{1}\right)$ is sampled from the base population to found a threatened population. After $t=N_{1}$ generations the population size can either be maintained $\left(N_{2}=N_{1}\right)$ or changed, and the population can enter, or not, a rescue program. Note that the time progresses downwards and that a waved edge is represented to indicate that the population was maintained with the same size before or after the time represented in the figure 
deleterious effect $s$ was sampled from a gamma distribution with mean $\bar{s}=0.2$ and shape parameter $\beta=0.33$, and the dominance coefficient $h$ was obtained from a uniform distribution between 0 and $\mathrm{e}^{(-k s)}$, where $k$ is such that the average $h$ value is $\bar{h}=0.283$, so that alleles that are more deleterious are expected to be more recessive (Caballero and Keightley 1994). Sampled $s$ values larger than 1 were assigned a value $s=1$ so that the mutational model generates a lethal class. Fitness was multiplicative across loci. The rationale for this mutational model is discussed below. In addition, neutral mutation was simulated to obtain estimates of neutral genetic diversity. One half of the individuals were assigned to each sex, and they were randomly chosen to breed according to their fitness and mated panmictically allowing for polygamy. A more detailed description is given in the Supplementary Material.

Figure 3 gives population fitness $w$ and inbreeding load $B$ averaged over replicates in a representative set of scenarios, always computed excluding migrants. Complementary results are given in the Supplementary Material. These results, analyzed in more detail in the main text, illustrate how, after the hybrid vigor occurred in the generations following migration events, some fitness rescue persists over generations when $N_{1} \leq N_{2}$ but a fitness disadvantage can be generated compared to "non-rescued" populations when $N_{1}>N_{2}$. It also shows that periodic migration induces strong fitness oscillations.

Figure 4 illustrates the between-population fitness variability introduced by periodic migration (upper panels) or OMPG (lower panels) for populations with sizes $N_{1}=50$, $N_{2}=4$. The panels on the left give the evolution of mean fitness for each of five randomly sampled populations under a non-rescue program; the panels on the right are for populations under rescue. Although in this 50-4 case periodic migration and OMPG increased long-term fitness if averaged over generations (Fig. 3), both strategies introduce important fitness variability between populations, which adds to the temporal oscillations in the case of periodic migration. This figure illustrates that every input of migrants in a small population can lead to a dangerous inbreeding depression after a few generations.

\section{End of Box 1}

Results in Fig. 3 give values averaged over replicates that illustrate the expected evolution of average fitness $(w)$ and inbreeding load $(B)$ over generations. They show that, under no rescue program, the expected fitness of threatened populations declined in the early generations and partially recovered a few generations later due to genetic purging. The decline was more dramatic and the recovery was poorer or non-existent in the smaller populations, as expected from less efficient purging. The inbreeding load also declined due to both genetic purging and drift. For the larger populations, this decline was much faster than that of the genetic diversity $(H)$ for neutral loci, due to efficient purging (Supplementary Material Figs. S1-S4). Finally, a new mutation-selection-drift balance was attained where $B$ depended just on $N_{2}$, while fitness depended on the size of the population through the whole period considered and continued to decline in the smaller populations due to the continuous fixation of deleterious mutations.

The introduction of migrants into the threatened populations always resulted in the increase of expected fitness (hybrid vigor) in the next generation, at the cost of an increase of the inbreeding load. Under occasional migration (one or two migration event), $B$ declined after this initial increase, approaching the same equilibrium values as those of populations under no rescue program. In contrast, under periodic migration and OMPG, $B$ oscillated around a plateau for values larger than those achieved under no rescue program.

The hybrid vigor after occasional migration was followed by new inbreeding depression to the point that, for $N_{1}>N_{2}$, where purging is more efficient before than after genetic flow, the expected fitness soon dropped to values persistently smaller than those of non-rescued lines (Fig. 3a).

Periodic migration every five generations produced a persistent rescue effect on expected fitness in all the same scenarios as occasional migration, as well as in all the cases with $N_{2}=4$ including those with $N_{1}>N_{2}$. However, it led to a strongly oscillatory behavior of the expected fitness (Fig. 3b and S3).

OMPG also improved expected fitness in all cases with the exception of 50-50 and 50-10, where it induced a slight disadvantage that still persisted by generation 100 (Fig. 3c and S4). The average advantages were stable through generations and were larger than under periodic migration.

These results are in general agreement with the qualitative predictions presented above (see Theoretical arguments). But, still, the main purpose in conservation genetics is not maximizing the expected average fitness but preventing population extinction (Bell et al. 2019). Figure 4 shows the evolution of average fitness for five randomly sampled non rescued lines and for five lines under periodic migration or OMPG for the 50-4 scenario. It illustrates that rescue events introduce temporal instability for the fitness of each line and increase the betweenlines fitness variance which, particularly under periodic migration, often leads to null or very small fitness values that would imply population extinction. Therefore, a positive effect of rescue on expected fitness does not imply a reduction in extinction risk. 
Fig. 3 Evolution of average fitness ( $w$; upper panels) and inbreeding load $(B$, lower panels) for endangered populations under different demographic and migration scenarios. Demographic scenarios are coded as $N_{1}-N_{2}, N_{1}$ indicating the population size during phase 1 , and $N_{2}$ during phase 2 (red, green and black lines for population sizes 4,10 or 50 , regardless the phase). Light dashed lines represent nonrescue lines. Dark solid lines represent lines entering a rescue program starting at generation $t=N_{1}$. a One unique migration of 5 males in lines $N_{2}=50$, and of 1 male otherwise. b Periodic migrations of 5 males every five generations in lines $N_{2}=50$, and of 1 individual otherwise. $\mathbf{c}$ One migrant male per generation (OMPG) a One migration

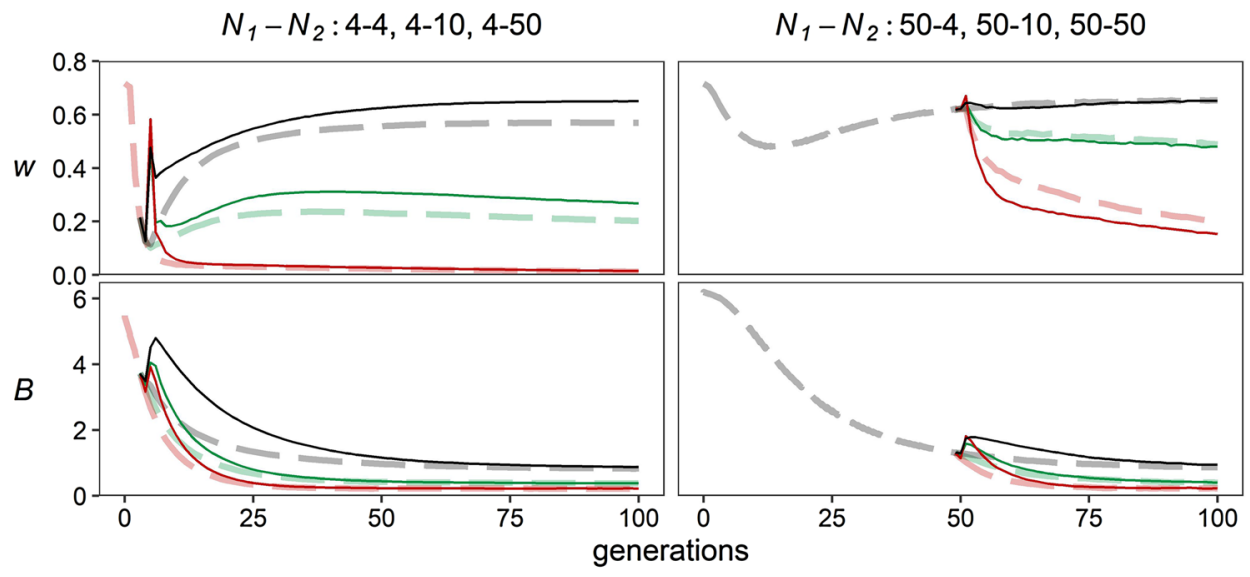

b Periodic migrations

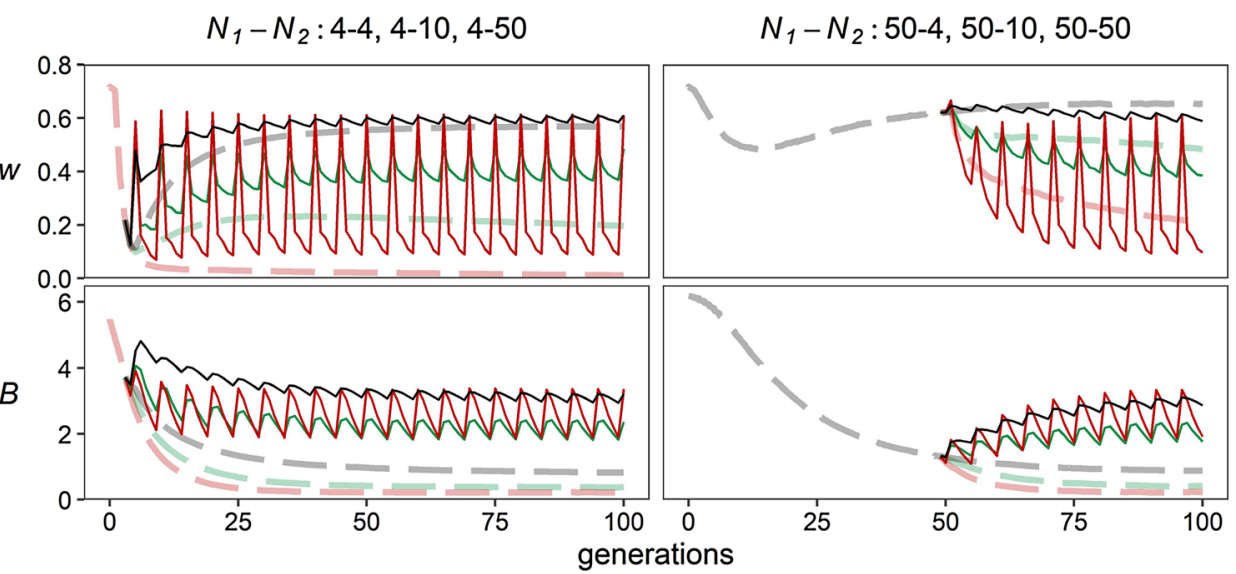

c OMPG

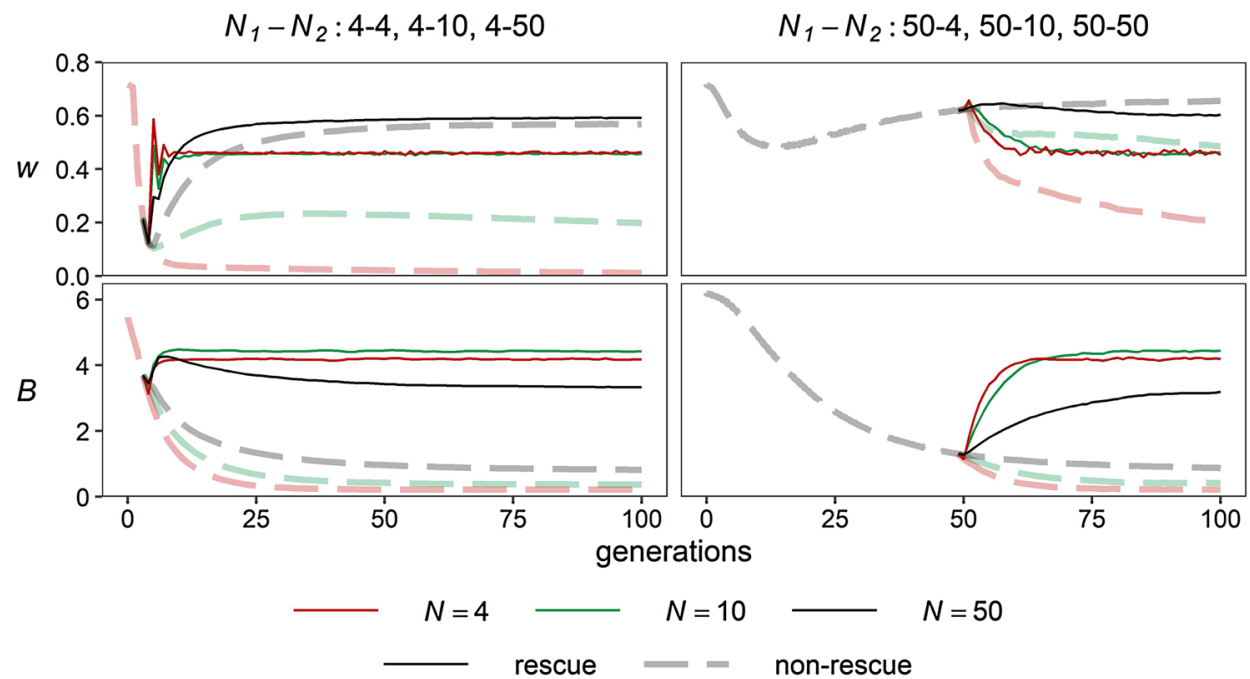

\section{Box 2: Consequences on population extinction}

Here we show extinction results corresponding to the scenarios described in Box 1. In these simulations, a population was considered extinct when the average fitness $(w)$ of males and/or females was less than 0.05 and/or when there were only breeding males or only breeding females (counted after migration when appropriate). Figure 5 shows the percentage of initial populations that survived through generations. 
Fig. 4 Genetic stochasticity for average fitness $(w)$ on sets of five random populations from those described in Fig. 3 for a scenario with population size $N_{1}=50$ during phase 1 and $\mathrm{N}_{2}=4$ during phase 2 (rescue starting at generation 50). Left panels: populations under no rescue program. Right panels: populations under a rescue program starting at generation 50 (upper panels for periodic migration every 5 generations, lower panels for OMPG)

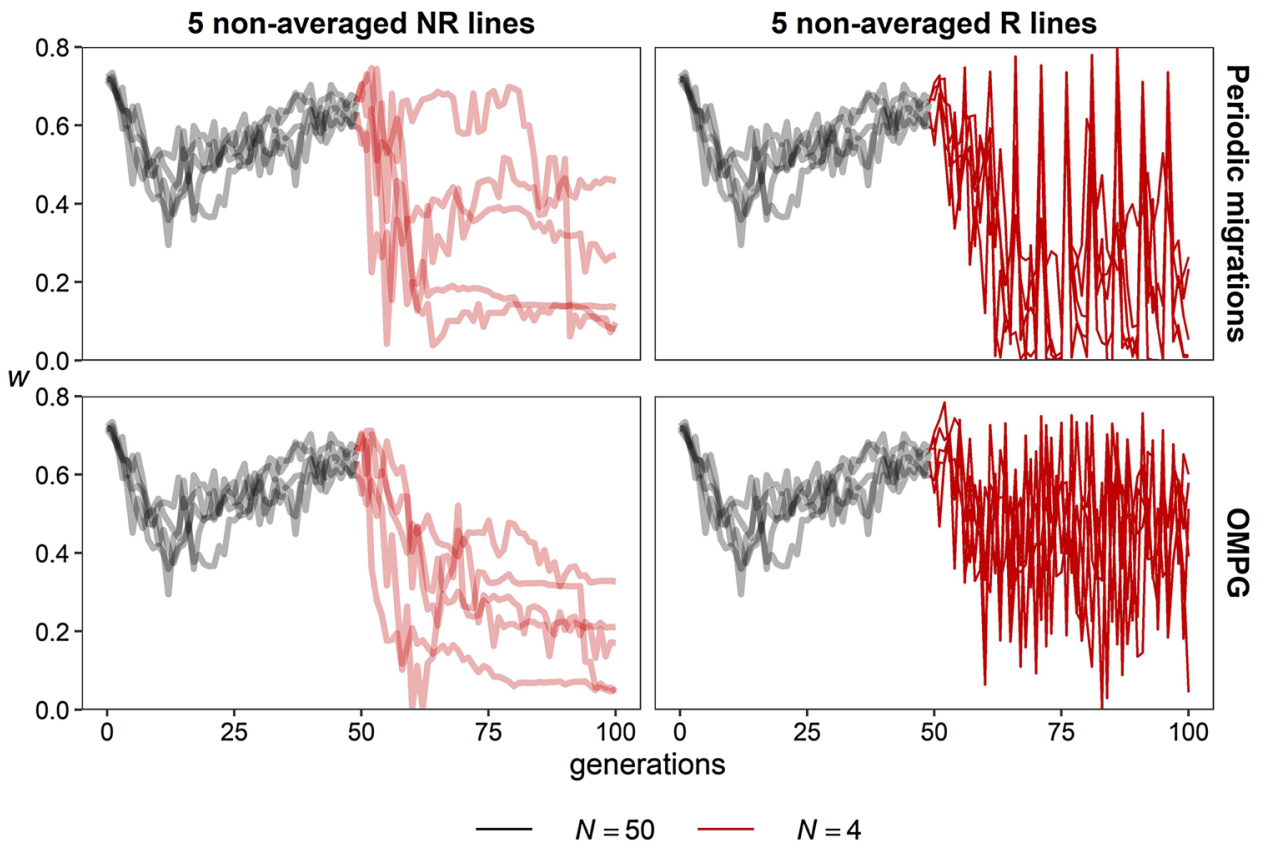

Genetic parameters averaged over surviving lines are given in Figs. S5-S8.

Under occasional migration, the rescue program increases the extinction risk in the cases where it induces a reduction of fitness compared to the non-rescue scenario (basically, when $\left.N_{1}>N_{2}\right)$. Periodic migration and OMPG increase the extinction risk of small populations $(N=4$ or 10$)$ in the medium or long term even in cases where they cause an increase of fitness averaged over generations. The reason for this increased extinction risk is the genetic stochasticity introduced by migration events, as illustrated in Fig. 4.

\section{End of Box 2}

Figure 5 gives simulation results illustrating the effect of rescue programs on population survival. In these simulations, occasional migration after a history of severe census decline produced a small but relevant reduction of the accumulated extinction risk when coupled with an increase of the population size (scenarios 4-10 and 4-50). However, for very small populations where purging had been more efficient previously (scenarios 10-4 and 50-4), occasional migration caused more extinction risk than no migration.

Except for the largest population sizes $\left(N_{2}=50\right)$ where no further extinction occurred, periodic migration or OMPG increased extinction risk in the medium to long term, the reduction being more dramatic under periodic migration. Both strategies caused an increased extinction risk even in scenarios where they cause higher expected fitness. The reason is the stochastic nature of the introduced load, a phenomenon already noted in other simulation analyses (Robert et al. 2003). Under periodic migration this stochasticity adds to the periodic oscillations of fitness, due to accelerated inbreeding depression following the initial hybrid vigor after migration. The OMPG strategy removes the periodic component (Fig. 4), which makes more likely to reduce extinctions during some time. In both cases, each migration event introduced randomly sampled deleterious alleles leading to occasional abrupt fitness declines in individual populations, which can boost the risk of extinction. The fact that successive migration events favor extinction in cases where a single or two events improved survival, suggests that extinction occurs due to the fortuitous accumulation of successive random fitness declines in the same population, each fueled by a migration event where the sampled migrants harbored large load. Additional extinction results under other extinction criteria are shown in Figs. S9-S10, giving similar results to those reported above.

\section{Discussion}

Our theoretical arguments and simulation results show that, considering a model of inbreeding load and genetic purging ascribed to partial recessive deleterious mutations, there are some specific situations where genetic rescue could be problematic. These situations are characterized by the use of migrants from non-purged donor populations that can introduce a substantial inbreeding load and genetic stochasticity into persistently small populations. The results suggest that additional caution needs to be introduced in the current genetic rescue paradigm (Ralls et al. 2018, 2020). 

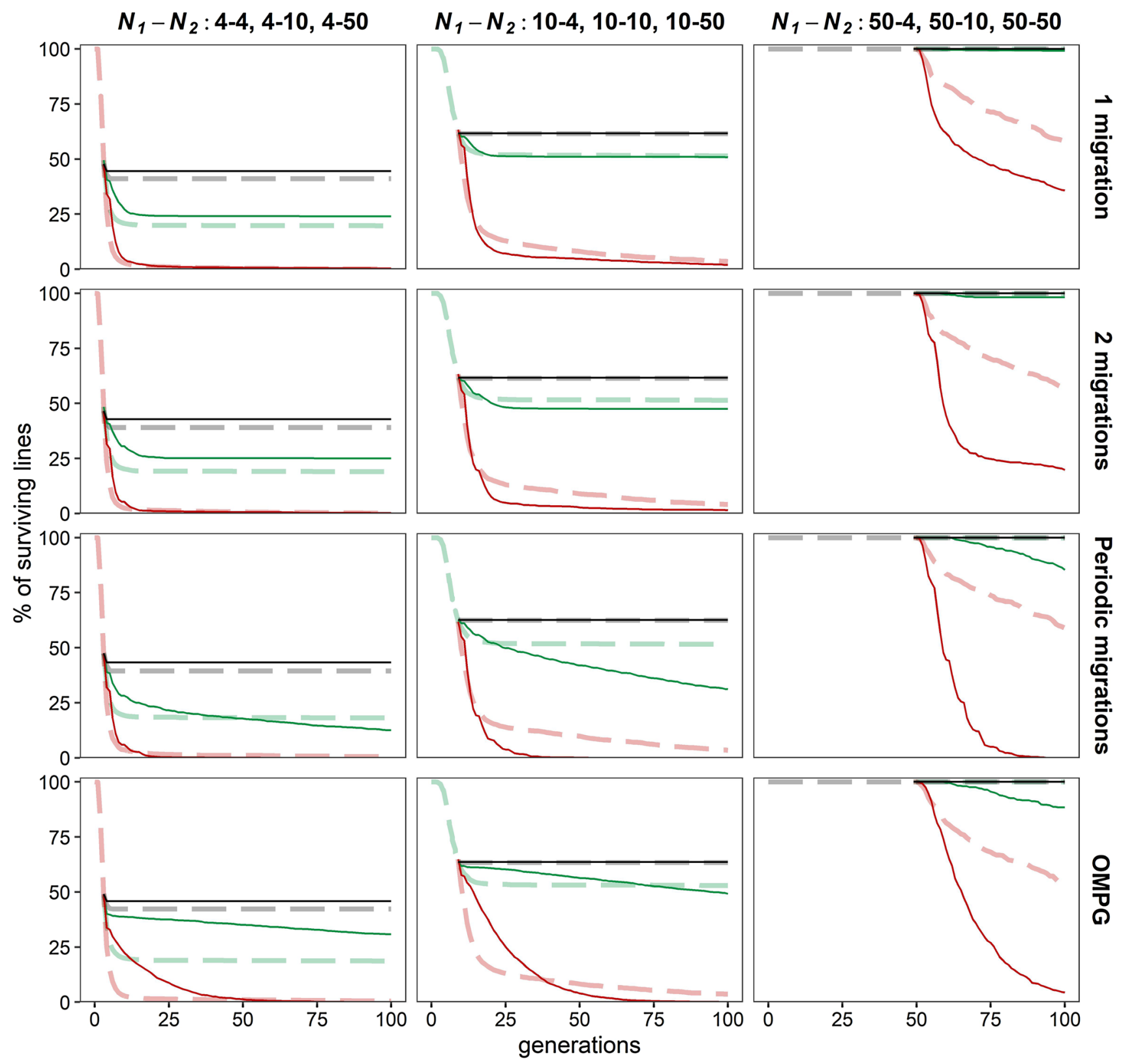

Fig. 5 Percentage of surviving populations through generations. Different rows of panels give results under a different migration scenario: one unique migration event; two migrations with an interval of five generations; periodic migrations every five generations; "one migrant per generation" strategy. Different columns are for different $N_{1}-N_{2}$

\section{Implications for conservation practice and some caveats of the simulation findings}

In practical situations, the relevance of purging on the outcome of a rescue program depends on many circumstances that have not been addressed here, such as demographic and demographic scenarios, coded as in Fig. 3 panels. Light dashed lines give the percentage of surviving populations under no rescue program while solid lines give results under the rescue program. Number of migrants per event as explained in Box 1

environmental stochasticity (particularly that affecting carrying capacity), adaptation to local conditions or the sex composition of migrants. Some of the factors not considered here can favor successful rescue. One main factor of hardly quantifiable consequences is the increased adaptive potential, which is favored by using large non-purged donor 
populations. Another possibly relevant factor is the introduction of favorable mutations occurred since the divergence between the donor and the recipient population, which are more likely to have accumulated in a large than in a small donor population (Ralls et al. 2020). This process of accumulation of new adaptive mutations is typically slow, so that it should not be relevant if the divergence is recent. On the contrary, if the divergence is more remote, the recipient population needs to have had large size during the majority of the divergence period in order to survive, so that it could have also accumulated different advantageous mutations, possibly implying some evolutionary divergence and risk of outbreeding depression (Edmands 2007). This is a subject that requires further investigation but, so far, there is little information available on the rate and nature of advantageous mutation for eukaryotes.

We have considered a model of inbreeding load $B$ ascribed to the recessive deleterious component of many rare detrimental alleles that remain hidden in the heterozygous condition in a non-inbred population. This is the most parsimonious explanation of inbreeding depression for which estimates of mutation rates and distribution of effects have been widely found empirically, and has repeatedly been consistent with the analysis of laboratory evolutionary experiments (see, e.g., Caballero 2020, Chap. 8). We have not considered overdominance, which may also contribute to inbreeding depression, but whose relative contribution is speculative and, according to most evidence, probably minor (Charlesworth and Charlesworth 1999; Charlesworth and Willis 2009; Hedrick 2012; Thurman and Barrett 2016). In a reanalysis of some available estimates of genetic variance components for viability in Drosophila melanogaster, Charlesworth (2015) concluded that balancing selection should partly explain the excess of variance observed in some populations for viability with respect to mutationselection balance (MSB) predictions. This conclusion, that could imply unrealistic low average viability in large Drosophila populations (i.e., high segregating load), is based on many crucial assumptions, including the supposition that $h$ is fully determined by $s$, or that the additive variance ascribed to recessive deleterious alleles at large populations corresponds to the MSB expectation and can be predicted in terms of the average $h$ value. However, the residual variability of $h$ conditional on $s$ can account for large amounts of inbreeding load and of variance, and recessive deleterious alleles can contribute substantially more additive variance in finite populations than expected at the MSB. No doubt that balancing selection due to antagonistic pleiotropy between fitness components can produce some excess in additive variance for viability (Fernández et al. 2005). However, an excess can also be expected in Drosophila due to pseudooverdominance generated by linked deleterious mutations (see Waller 2021, for a recent study on the subject), because of the reduced length of the genome and its multiple inversions, as well as to genotype-environment interactions, as suggested by Mukai (1988) and Santos (1997). Contrary to the result of Charlesworth (2015), Sharp and Agrawal (2018) found no excess of variance for viability in laboratory Drosophila populations when compared to expected values computed using the decline of average viability in mutation accumulation lines. Although there was an excess of variance with respect to MSB predictions for fecundity and male mating success, it should be interpreted with caution due to (i) natural selection possibly biasing the estimate of mutational mean decline in the mutation accumulation lines; b) possible differences between the magnitude of effects expressed during traits' assay protocol and during population maintenance, as noted by the authors. In what follows, we concentrate on the consequences on rescue of the inbreeding load ascribed to deleterious alleles, and we leave the consequences of overdominant loci to be explored in the future in cases where it might be proven to be relevant.

In our simulations, migrant individuals were always males, but results would have been the same if migrants had been all females, because the distribution of the number of matings per individual, as well as that of the number of offspring contributed to the next generation, were the same for both sexes. However, in practical cases, using just female migrants can allow a better control of the amount and variability of inbreeding load introduced, while using males with a mating advantage can boost the short-term demographic rescue (Zajitschek et al. 2009) but also the spread of the immigrant's inbreeding load, as in the case of the wolf's population of Isle Royale (Hedrick et al. 2014, 2017, 2019).

A consequence of all migrants having the same sex is that they always mate individuals of the endangered population. Therefore, a maximum hybrid vigor is expected in the first generation, but half of it would be expected to be lost in the absence of selection after one generation of panmixia. In the real world, the existence of maternal genetic effects on fitness may add a delayed component for hybrid vigor and inbreeding depression, obscuring the temporal fitness profile (Caballero 2020, p. 197). Therefore, the importance of the sex of migrants and of genetic maternal components on the dynamics of hybrid vigor and inbreeding load under repeated migration deserve being investigated.

Our finding of increased extinction risk for small populations under some scenarios of rescue programs seems to be in contradiction with the quite general view that, after excluding cases where outbreeding depression was to be expected, introduction of migrants always causes successful genetic rescue, usually assayed in terms of improved fitness or population sizes (Waller 2015; Frankham 2015, 2016; Ralls et al. 2020). However, evaluating genetic rescue effects is not simple (Robinson et al. 2020), and this view is grounded on rescue programs that had been tracked for 


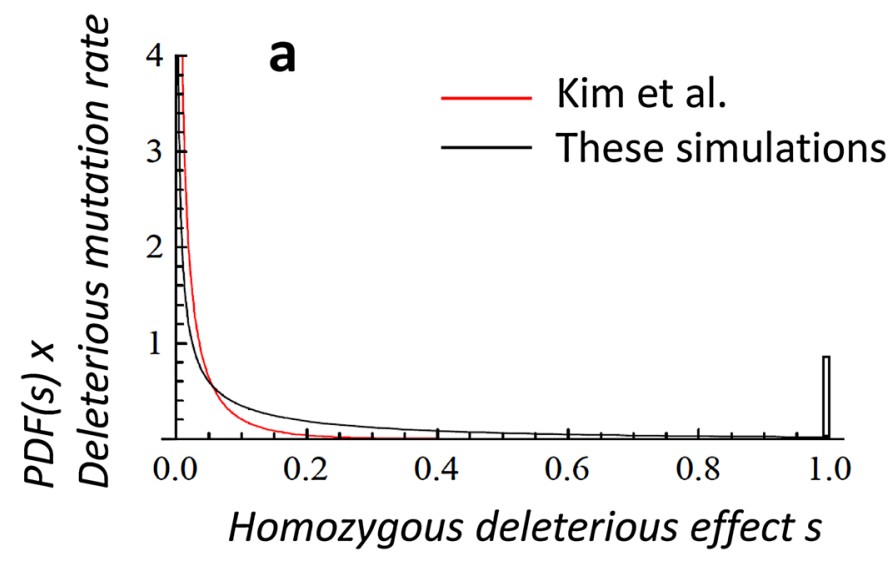

Fig. 6 Mutational models. a Probability density function (PDF) of the homozygous deleterious mutational effects multiplied by the deleterious mutation rate. Red line: Model inferred from evolutionary genomic analysis by Kim et al. (2017) (best fit model for the 1000 genomes data: mutation rate per gamete and generation 0.314 , homozygous effect $s$ gamma distributed with shape parameter 0.186 and mean 0.0161 , predicted equilibrium inbreeding load $B=3.07$ for effective size $10^{4}$ ). Black line: Model used in our simulations (mutation rate per gamete and generation $0.2, s$ gamma distributed with

just a few generations after immigration events (usually 1-3 generations, 5-6 on a few occasions) or on hybridization of populations (Chan et al. 2018), which is not the common situation in genetic rescue programs (Whiteley et al. 2015).

Our results are in disagreement in some respects with the simulation results obtained by Kyriazis et al. (2020), who found a substantial extinction rate for endangered populations with $N_{e}=50$ (or 25) which was always reduced under rescue programs. There are two main differences between the simulations by Kyriazis et al. (2020) and those presented here that could explain these disagreements. One is that they chose to simulate more realistic scenarios from an ecological point of view, in order to assess the joint consequences of genetic and nongenetic factors. We chose to illustrate the relevance of purging in simplified ecological conditions, an approach that allows a clearer understanding of the main genetic processes but leaves unexplored the relevance of their interaction with ecological factors. This could explain the larger extinction risks observed by Kyriazis et al. (2020). However, the different findings regarding the success of genetic rescue to prevent extinction is more likely to be due to the different mutational models used. Kyriazis et al. (2020), following a recent accepted trend, take the mutational model from estimates based on the evolutionary analysis of genomic data on site frequency spectra (Kim et al. 2017), which are very sensitive to the distribution of mild deleterious effects $(s<0.02$ in homozygosis) but quite insensitive to the differences in deleterious effects above this threshold, which are conceptually pooled into a single "strongly deleterious" effect class. The problem is

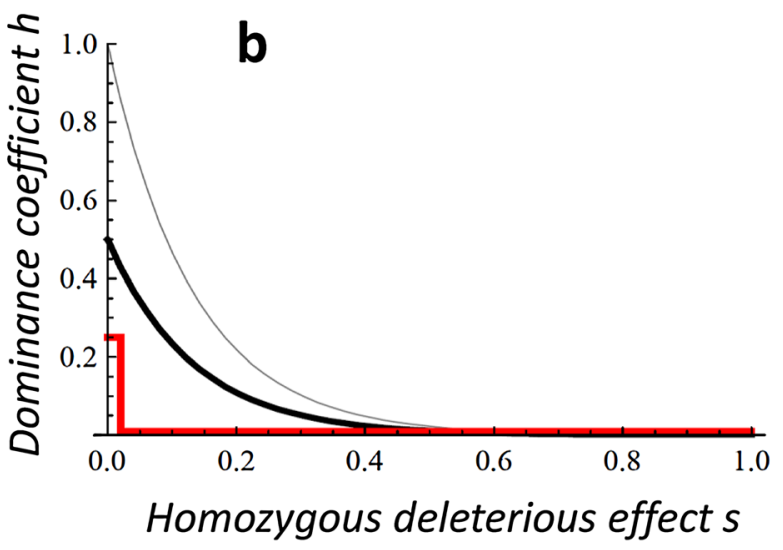

shape parameter 0.33 and mean 0.2 , predicted equilibrium inbreeding load $B=6.3$ for effective size $10^{4}$ ); the lethal class generated in this model by assigning $s=1$ to $s$ values above 1 is represented in the [0.99-1] interval. b The black thick line gives the average inbreeding coefficient as a function of $s$ assumed in our simulations, where $h$ is uniformly distributed between 0 and the thin black line (extracted from García-Dorado and Caballero 2000 and García-Dorado 2003). The red line gives the $h$ values used by Kyriazis et al. (2020) simulations, which are constant for each value of $s$

that, under these mutational models, the rate of mutations with $s>0.1$ is tiny (Fig. 6a). However, there is evidence that a large fraction of the inbreeding depression that compromises the survival of endangered populations is due to large deleterious effects spread in the interval $(0.1,1]$, including lethals (Caballero and Keightley 1998; Bijlsma et al. 1999; García-Dorado et al. 2007; Fox et al. 2008; Charlesworth and Willis 2009; Hedrick et al. 2016; Domínguez-García et al. 2019). In addition, despite using a distribution of deleterious effects inferred assuming additivity, non-additive gene action is simulated. This is achieved by using a model equivalent to assigning $h=0.25$ to any deleterious mutation with $s<0.02$ and assigning $h=0$ for $s \geq 0.02$. This model is not consistent with inferences from classical experiments according to which deleterious mutations with $s \geq 0.02$ are on the average only partially recessive (García-Dorado and Caballero 2000; Halligan and Keightley 2009; Ralls et al. 2020), as illustrated in Fig. 6b. Under this mutational model, the inbreeding load concealed in each individual is ascribed to very many recessive deleterious alleles, each with a very small effect. Therefore, the coefficient of variation of the inbreeding load introduced by migrant individuals is very small, and the corresponding extinction risk ascribed to the genetic stochasticity is negligible.

In our simulations, we used a deleterious mutation rate and a joint distribution of $s$ and $h$ chosen to jointly account for: (a) the large rate of deleterious mutations unveiled by the evolutionary analysis of genomic data, with prevailing mild deleterious effect $s<0.01$ that are likely to be roughly additive (Keightley and Eyre-Walker 2007; Boyko et al. 
2008; Kim et al. 2017); (b) the results from classical mutation accumulation and fitness assay experiments, which are unlikely to detect small deleterious effects (say, $s<10^{-3}$ ) but imply a relevant rate of deleterious mutations with effects $s>0.1$ that are severe from a conservation point of view (García-Dorado 1997; Caballero et al. 2002; Halligan and Keightley 2009; Thurman and Barrett 2016) and whose average degree of dominance is inversely related to their deleterious effect (García-Dorado and Caballero 2000; GarcíaDorado 2003); (c) the large inbreeding load concealed in large wild populations: under our deleterious mutation model, the expected haploid inbreeding load $B$ for an equilibrium population with effective size $N=10^{4}$, computed by integrating the equation for the equilibrium inbreeding load (García-Dorado 2007) into the joint distribution for $s$ and $h$ was $B=6.23$ (1.885 for lethal alleles), which is on the order of that found in several wild populations of mammals and birds (O'Grady et al. 2006; Hedrick and García-Dorado 2016); (d) The relatively large efficiency of genetic purging obtained from appropriate data under moderate bottlenecking (Bersabé and García-Dorado 2013; López-Cortegano et al. 2016). Figure 6a illustrates that, under this model, the small rate of mutation with deleterious alleles that are severe in the conservation context (say, $s>0.1$ ), is much larger than that assumed under the mutational model inferred from genomic evolutionary analysis. In our model, the dominance coefficient (Fig. 6b) is not completely determined by the homozygous deleterious effect although, according to empirical evidence, its expected value progressively decays with increasing $s$, so that most semilethal mutations are virtually recessive. Our knowledge on the joint distribution of mutational deleterious effects and dominance coefficients, including the spectra of severely deleterious effects above 0.1 , is quite limited for species of conservation concern, so more information is needed in this respect. In any case, our results illustrate that it is necessary to be cautious, since the inbreeding load introduced by migrants from large nonpurged populations can have an important sampling variance and the potential to compromise population survival depending on the demographic past and future of the endangered population.

\section{Considering the difference between reconnection and occasional or recurrent rescue}

Our simulation results illustrate that continuous stable reconnection is safer than occasional or recurrent migration events. Furthermore, an important feature of reconnection in the wild is that population extirpation can in principle be reverted by recolonization, so that extinction should be referred to the metapopulation (Brown and Kodric-Brown 1977; Eriksson et al. 2014). Therefore, in this context, our simulation results for the evolution of fitness average under
OMPG are more relevant than those for population survival which did not account for recolonization. Those fitness results suggest that continuous connection should improve the persistence of small populations. In practice, after restoring connectivity between the endangered population and a larger population or a metapopulation, the endangered population can enter an extirpation-recolonization dynamic that depends on many demographic and ecological factors (Franken and Hik 2004), as well as on the genetic stochasticity arising from the migration pattern. Then, an equilibrium is expected in the long term where the genetic identity of each population is scarcely affected by historical extirpationrecolonization events. In such a situation, a stable but limited connectivity (e.g., OMPG) can allow enough inbreeding from partial fragmentation to promote some purging, while preventing both the further progress of inbreeding depression and the metapopultion extinction.

Therefore, it is convenient to establish a conceptual distinction between genetic rescue programs based on occasional or even periodic migration, and programs aiming the continuous stable connection between the endangered population and a large healthy one (or a metapopulation). The former could be considered "sensu stricto rescue" programs, in the sense that they are intended to avoid the extinction of an isolated population whose stable reconnection is not feasible or whose differentiated genetic identity is worth to be preserved, although they involve some risk of swamping such identity. The second ones, say "reconnection rescue" programs, here represented by what might be considered its minimum migration rate (OMPG), may rather be aimed to preserve the endangered population as one of the valuable pieces integrating a metapopulation and the whole ecosystem, but one that does not show distinctive genetic features or adaptations to be preserved. This reconnection could be achieved either by actively moving individuals on a regular basis or by reconnecting landscapes, which has the advantage of setting an autonomous non-assisted mechanism. Besides allowing recolonization after extirpation, reconnecting landscapes will allow bidirectional flow, shifting the conservation aim from avoiding extinction of the endangered subpopulation to improving its long-term expected contribution to the metapopulation survival.

\section{The relevance of purging to rescue success under different conservation scenarios}

The preceding sections show that, before introducing migrants from a large population into a critically endangered one, we should analyze the prospects that the increased reproductive potential expected from hybrid vigor immediately after migration and the habitat availability will allow the population to recover at least a moderate effective size in the near future, as in the case of Scandinavian wolves (Vilà 
et al. 2003). Furthermore, it is also convenient to gather information on the demographic history of the donor and the recipient population so that we can infer whether they underwent efficient purging in the past, as well as on the possibility that a stable reconnection can be achieved. To illustrate how our results can be considered to assist decisions regarding the introduction of migrants from a large non-purged population into an endangered one, below we present three different representative simplified scenarios.

(i) In the first scenario, we consider the introduction of migrants to restore adaptive potential in an endangered population that has persisted for a long time with a moderate effective size (50 or above). In this case, the main purpose is not to reduce inbreeding depression or to ameliorate the load accumulated from continuous deleterious mutation, as both should be small due to past efficient purging (as well as to non-purging selection). Furthermore, if the population size does not decline further, purging is also expected to remove the load contributed by migrants, so that the genetic rescue program is not expected to be crucial by reducing inbreeding depression. In this situation, the main purpose of the rescue program is to restore adaptive potential and to prevent long term risk due to fixation of new deleterious mutation. Note, however, that inducing gene flow in populations that have survived for a very long time with moderate size and whose reproductive potential is large enough to allow population persistency may imply an unjustified risk, as has been appreciated in the case of Island foxes (Robinson et al. 2018). In particular, it could increase their inbreeding depression in case of future inbreeding. Special consideration deserves the case of endangered populations that have evolved differential adaptation or distinctive features, which could be swamped due to introgression or could lead to outbreeding depression (Hedrick and Fredrickson 2010; Frankham et al. 2011; Harris et al. 2019). However, there is some evidence that natural selection favoring locally adaptive alleles can efficiently prevent introgression of mis-adapted alleles during genetic rescue (Fitzpatrick et al. 2020).

(ii) In the second scenario, the rescue program is intended to restore both fitness and genetic diversity in a population that gets over a critical period of very small effective size $\left(N_{e}<10\right)$ but has now recovered to some degree or is expected to do so quickly. During the past bottleneck, this population underwent high inbreeding, low purging and, therefore, an important reduction of fitness and of adaptive potential. The rescue program is expected to produce an immediate hybrid vigor and to provide wild alternatives to the deleterious alleles that might have been previously fixed in the endangered population. If the effective population size is moderately large at present (50 or above under our mutational model), or is expected to be so soon after receiving migrants, natural selection is expected to favor these introduced wild variants and to, slowly but efficiently, purge introduced recessive deleterious ones. And, of course, immigration will help to restore genetic diversity. Therefore, a rescue program integrated with measures that prompt early demographic restoration is expected to be helpful both in the short and the long term (Hufbauer et al. 2015). If the population recovery is less prosperous, one or two migratory events may be helpful to reduce the extinction risk before purging restores the population fitness, but periodic migration, advised in order to prevent the progressive loss of genetic diversity (Miller et al. 2020), could increase the extinction risk in the long term due to random accumulation of migration events introducing large amounts of inbreeding load. Then, a compromise should be achieved by favoring donor populations that are expected to be purged but that still contribute to enrich the genetic diversity of the recipient one.

(iii) Finally, the third scenario corresponds to rather extreme situations within the unfortunately paradigmatic case of an isolated population whose size has progressively declined over time, often beginning with a period where the decline was cryptic. With such demographic history, the ancestral inbreeding load should have been purged to a considerable extent. At present, the habitat has been reduced or degraded to a point that the effective size is dramatically small and is unlikely to grow in the near future. In such a situation, each migration event with individuals sampled from a large non-purged population produces some increase in mean fitness, but can be followed by accelerated inbreeding depression, increased stochasticity for fitness average, and increased extinction risk. The reason is that, due to the purging occurred while the size of the endangered population was moderate, the inbreeding load introduced by migrant individuals can be larger than the overall deleterious load of resident ones. Furthermore, the introduced load is boosted by the fitness advantage of the migrants and their vigorous crossed offspring (Saccheri and Brakefield 2002; Bijlsma et al. 2010), and it is inefficiently purged due to the small effective size. In these cases, periodically repeated rescue cycles allow the fortuitous concatenation of migration events introducing too high inbreeding load, worsening the survival prospects. 
Note that we are considering that, although the initial increase of fitness is expected to improve the intrinsic growth rate, the population size will continue to be small due to the limiting environmental factors and carrying capacity.

This scenario (iii) is vividly illustrated by the extinction of the Isle Royale wolves population, occurred after a sudden fitness increase caused by a single migrant from the continental population (Hedrick et al. 2019; Robinson et al. 2019). This scenario may seem of limited practical interest, as it refers to a very small effective population size. However, in wild populations, the effective size is usually much smaller than the actual number of breeding adults (Frankham 1995; Vucetich et al. 1997; Palstra and Ruzzante 2008). Furthermore, the effective size of many endangered populations has progressively declined down to values on the order of tens, as in the cases the Chatham Island black robin Petroica traversi (Ardern and Lambert 1997; Weiser et al. 2016) or the Fennoscandian arctic fox Vulpes lagopus (Angerbjörn et al. 2013; Norén et al. 2016), and many others. In some of these cases, population growth is limited by non-genetic factors, as habitat or prey limitations (Adams et al. 2011; Hedrick et al. 2014). Population growth can also be limited by inbreeding depression that could later be reverted to some extent due to genetic purging. Thus, this scenario, although extreme, can cover some practical conservation cases. Nevertheless, the specific risks in each situation are unknown due to the uncertainties surrounding the rate and effect distribution of deleterious mutation or the demographic history of populations, as well as to the many other genetic and non-genetic factors discussed above.

It is true that there is a pressure for taking action regarding critically endangered populations (Ralls et al. 2018), as their medium-term persistence is critically compromised if only due to demographic stochasticity. However, these results show that rescue interventions in persistently small populations may increase their long term extinction risk in some cases, calling for additional caution. In such cases, the rescue program should be coupled with reinforced habitat interventions in order to restore an effective population size large enough to allow efficient purging. Note that if, due to past purging under slow inbreeding, the endangered population shows no evidence of inbreeding depression, the rescue program is mainly intended to restore adaptive potential and might be postponed until a larger size is attained. Alternatively, one or several donor populations with a history of slow inbreeding, and therefore more purged, could be preferred, although the decision should weight the risk arisen from the introduced load with others derived from causes not included in these simulations, as the loss of adaptive potential. In any case, as far as the population singularity is not a main concern, the restoration of continuous connectivity should be preferred to recurrent migration events from time to time, due to fortuitous accumulation of random episodes introducing high load.

\section{Future advances and conclusions}

The impact on conservation practice of the theoretical considerations and the simulation results discussed here depend on many factors that determine the genetic architecture of the inbreeding load, as the distribution of mutational effects and dominance patterns for deleterious mutations or the complexity of demographic histories, and all of them are worthwhile to be explored. Simulation approaches and experiments with model organisms can be very useful, both to advance in our understanding of these genetic features and to test the predictions generated in this analysis. It would also be helpful to understand how the genomic load assayed in terms of the burden of alleles annotated for different deleterious categories can inform on the fitness load and, in particular, on the inbreeding load measured in terms of concealed deleterious effects. Furthermore, there is a need for long term empirical observation of case studies, based on careful evaluation of the inbreeding load and the demographic and genetic flow history in both the donor and the recipient population, the evolution of fitness in the latter and the occurrence of extinction. These studies could benefit on the combined assay of fitness traits and genomic information.

The prospects of a rescue program depend on the demographic history of the endangered and donor populations but, in agreement with the small population paradigm, future population growth is essential to guarantee successful rescue and improve population survival. Our results illustrate that understanding all the consequences of conservation interventions is an arduous enterprise riddled with difficulties, and that the only safe strategy for in situ conservation and the one that should be prioritized and taken as a paradigm, is the recovery of large effective population size through the restoration of the habitat and of a healthy and continuous connectivity.

Supplementary Information The online version contains supplementary material available at https://doi.org/10.1007/s10592-021-01405-7.

Acknowledgements We are grateful to the editor and to three anonymous reviewers by their helpful comments.

Funding This work was funded by Agencia Estatal de Investigación (AEI) (PGC2018-095810-B-I00 and PID2020-114426 GB-C21), Xunta de Galicia (GRC, ED431C 2020-05) and Centro singular de investigación de Galicia accreditation 2019-2022, and the European Union (European Regional Development Fund-ERDF), Fondos Feder "Unha maneira de facer Europa". N.P.-P. is funded by a predoctoral (FPU) grant from Ministerio de Educación, Cultura y Deporte (Spain). 
Data availability Not applicable.

Code availability Codes will be available at GitHub address https:// github.com/noeliaperezp/Genetic_Rescue.

\section{Declarations}

Conflict of interest Not applicable.

Ethical approval Not applicable.

Consent to participate Not applicable.

Consent for publication All authors have approved the manuscript for publication.

Open Access This article is licensed under a Creative Commons Attribution 4.0 International License, which permits use, sharing, adaptation, distribution and reproduction in any medium or format, as long as you give appropriate credit to the original author(s) and the source, provide a link to the Creative Commons licence, and indicate if changes were made. The images or other third party material in this article are included in the article's Creative Commons licence, unless indicated otherwise in a credit line to the material. If material is not included in the article's Creative Commons licence and your intended use is not permitted by statutory regulation or exceeds the permitted use, you will need to obtain permission directly from the copyright holder. To view a copy of this licence, visit http://creativecommons.org/licenses/by/4.0/.

\section{References}

Adams JR, Vucetich LM, Hedrick PW, Peterson RO, Vucetich JA (2011) Genomic sweep and potential genetic rescue during limiting environmental conditions in an isolated wolf population. Proc R Soc B 278:3336-3344. https://doi.org/10.1098/rspb.2011.0261

Åkesson M, Liberg O, Sand H, Wabakken P, Bensch S, Flagstad Ø (2016) Genetic rescue in a severely inbred wolf population. Mol Ecol 25:4745-4756. https://doi.org/10.1111/mec.13797

Allendorf FW, Luikart G, Aitken SN (2013) Conservation and the genetics of populations. 2nd edn. Wiley-Blackwell, West Sussex.

Angerbjörn A, Eide NE, Dalén L, Elmhagen B, Hellström P, Ims RA, Killengreen S, Landa A, Meijer T, Mela M, Niemimaa J, Norén K, Tannerfeldt M, Yoccoz NG, Henttonen H (2013) Carnivore conservation in practice: replicated management actions on a large spatial scale. J Appl Ecol 50:59-67. https://doi.org/10. 1111/1365-2664.12033

Ardern SL, Lambert DM (1997) Is the black robin in genetic peril? Mol Ecol 6:21-28. https://doi.org/10.1046/j.1365-294X.1997. 00147.x

Ávila V, Amador C, García-Dorado A (2010) The purge of genetic load through restricted panmixia in a Drosophila experiment. J Evol Biol 23:1937-1946. https://doi.org/10.1111/j.1420-9101. 2010.02058.x

Bell DA, Robinson ZL, Funk WC, Fitzpatrick SW, Allendorf FW, Tallmon DA, Whiteley AR (2019) The exciting potential and remaining uncertainties of genetic rescue. Trends Ecol Evol 34:1070-1079. https://doi.org/10.1016/j.tree.2019.06.006

Bersabé D, García-Dorado A (2013) On the genetic parameter determining the efficiency of purging: an estimate for Drosophila eggto-pupae viability. J Evol Biol 26:375-385. https://doi.org/10. $1111 /$ jeb. 12054
Bijlsma R, Bundgaard J, Van Putten WF (1999) Environmental dependence of inbreeding depression and purging in Drosophila melanogaster. J Evol Biol 12:1125-1137. https://doi. org/10.1046/j.1420-9101.1999.00113.x

Bijlsma R, Westerhof MDD, Roekx LP, Pen I (2010) Dynamics of genetic rescue in inbred Drosophila melanogaster populations. Conserv Genet 11:449-462. https://doi.org/10.1007/ s10592-010-0058-z

Boyko AR, Williamson SH, Indap AR, Degenhardt JD, Hernandez RD, Lohmueller KE, Adams MD, Schmidt S, Sninsky JJ, Sunyaev SR, White TJ, Nielsen R, Clark AG, Bustamante CD (2008) Assessing the evolutionary impact of amino acid mutations in the human genome. PLoS Genet 4:e1000083. https:// doi.org/10.1371/journal.pgen.1000083

Brown JH, Kodric-Brown A (1977) Turnover rates in insular biogeography: effect of immigration on extinction. Ecology 58:445449. https://doi.org/10.2307/1935620

Caballero A (2020) Quantitative genetics. Cambridge University Press, Cambridge

Caballero A, Keightley PD (1994) A pleiotropic nonadditive model of variation in quantitative traits. Genetics 138:883-900

Caballero A, Keightley PD (1998) Inferences on genome-wide deleterious mutation rates in inbred populations of Drosophila and mice. Genetica 102(103):229-239. https://doi.org/10.1007/ 978-94-011-5210-5_19

Caballero A, Cusi E, Garcia C, García-Dorado A (2002) Accumulation of deleterious mutations: additional Drosophila melanogaster estimates and a simulation of the effects of selection. Evolution 56:1150-1159. https://doi.org/10.1111/j.0014-3820. 2002.tb01428.x

Caballero A, Bravo I, Wang J (2017) Inbreeding load and purging: implications for the short-term survival and the conservation management of small populations. Heredity 118:177-185. https://doi.org/10.1038/hdy.2016.80

Caballero A, Villanueva B, Druet T (2020) On the estimation of inbreeding depression using different measures of inbreeding from molecular markers. Evol Appl. https://doi.org/10.1111/ eva.13126

Chan WY, Hoffmann AA, van Oppen MJH (2018) Hybridization as a conservation management tool. Conserv Lett 12:e12652. https://doi.org/10.1111/conl.12652

Charlesworth B (2015) Causes of natural variation in fitness: evidence from studies of Drosophila populations. Proc Natl Acad Sci USA 112:1662-1669. https://doi.org/10.1073/pnas.14232 75112

Charlesworth B, Charlesworth D (1999) The genetic basis of inbreeding depression. Genet Res Camb 74:329-340. https://doi.org/10. 1017/S0016672399004152

Charlesworth D, Willis JH (2009) The genetics of inbreeding depression. Nat Rev Genet 10:783-796. https://doi.org/10.1038/nrg26 64

Domínguez-García S, García C, Quesada H, Caballero A (2019) Accelerated inbreeding depression suggests synergistic epistasis for deleterious mutations in Drosophila melanogaster. Heredity 123:709-722. https://doi.org/10.1038/s41437-019-0263-6

Edmands S (2007) Between a rock and a hard place: evaluating the relative risks of inbreeding and outbreeding for conservation and management. Mol Ecol 16:463-475

Eriksson A, Elías-Wolff F, Mehlig B, Manica A (2014) The emergence of the rescue effect from explicit within-and between-patch dynamics in a metapopulation. Proc R Soc B 281:20133127. https://doi.org/10.1098/rspb.2013.3127

Falconer DS, Mackay TFC (1996) Introduction to quantitative genetics. Longman, Essex

Fernández B, García-Dorado A, Caballero A (2005) The effect of antagonistic pleiotropy on the estimation of the average 
coefficient of dominance of deleterious mutations. Genetics 171:2097-2112

Fitzpatrick SW, Bradburd GS, Kremer CT, Salerno PE, Angeloni LM, Funk WC (2020) Genomic and fitness consequences of genetic rescue in wild populations. Curr Biol 30:517-522. https://doi.org/10.1016/j.cub.2019.11.062

Franken RJ, Hik DS (2004) Influence of habitat quality, patch size and connectivity on colonization and extinction dynamics of collared pikas Ochotona collaris. J Anim Ecol 73:889-896. https://doi.org/10.1111/j.0021-8790.2004.00865.x

Frankham R (1995) Effective population size/adult population size ratios in wildlife: a review. Genet Res 66:95-107. https://doi. org/10.1017/S0016672300034455

Frankham R (2015) Genetic rescue of small inbred populations: metaanalysis reveals large and consistent benefits of gene flow. Mol Ecol 24:2610-2618. https://doi.org/10.1111/mec.13139

Frankham R (2016) Genetic rescue benefits persist to at least the F3 generation, based on a meta-analysis. Biol Conserv 195:33-36. https://doi.org/10.1016/j.biocon.2015.12.038

Frankham R, Ballou JD, Eldridge MD, Lacy RC, Ralls K, Dudash MR, Fenster CB (2011) Predicting the probability of outbreeding depression. Conserv Biol 25:465-475. https://doi.org/10. 1111/j.1523-1739.2011.01662.x

Frankham R, Bradshaw CJ, Brook BW (2014) Genetics in conservation management: revised recommendations for the 50/500 rules, Red List criteria and population viability analyses. Biol Conserv 170:56-63. https://doi.org/10.1016/j.biocon.2013.12. 036

Fredrickson RJ, Siminski P, Woolf M, Hedrick PW (2007) Genetic rescue and inbreeding depression in Mexican wolves. Proc R Soc B 274:2365-2371. https://doi.org/10.1098/rspb.2007.0785

Fox CW, Scheibly KL, Reed DH (2008) Experimental evolution of the genetic load and its implications for the genetic basis of inbreeding depression. Evolution 62:2236-2249. https://doi.org/10. 1111/j.1558-5646.2008.00441.x

García-Dorado A (1997) The rate and effects distribution of viability mutation in Drosophila: minimum distance estimation. Evolution 51:1130-1139. https://doi.org/10.1111/j.1558-5646.1997. tb03960.x

García-Dorado A (2003) Tolerant versus sensitive genomes: the impact of deleterious mutation on fitness and conservation. Conserv Genet 4:311-324. https://doi.org/10.1023/A:1024029432658

García-Dorado A (2007) Shortcut predictions for fitness properties at the MSD balance and for its build-up after size reduction under different management strategies. Genetics 176:983-997. https:// doi.org/10.1534/genetics.106.065730

García-Dorado A (2012) Understanding and predicting the fitness decline of shrunk populations: inbreeding, purging, mutation, and standard selection. Genetics 190:1461-1476. https://doi.org/ 10.1534/genetics.111.135541

García-Dorado A, Caballero A (2000) On the average coefficient of dominance of deleterious spontaneous mutations. Genetics 155:1991-2001

García-Dorado A, Caballero A (2021) Neutral genetic diversity as a useful tool for conservation biology. Conserv Genet 22:541-545. https://doi.org/10.1007/s10592-021-01384-9

García-Dorado A, Ávila V, Sánchez-Molano E, Manrique A, LópezFanjul C (2007) The build up of mutation-selection-drift balance in laboratory Drosophila populations. Evolution 61:653-665. https://doi.org/10.1111/j.1558-5646.2007.00052.x

Grossen C, Guillaume F, Keller LF, Croll D (2020) Purging of highly deleterious mutations through severe bottlenecks in Alpine ibex. Nat Commun 11:1001. https://doi.org/10.1038/ s41467-020-14803-1

Halligan DL, Keightley PD (2009) Spontaneous mutation accumulation studies in evolutionary genetics. Annu Rev Ecol Evol Syst
40:151-172. https://doi.org/10.1146/annurev.ecolsys.39.110707. 173437

Harris K, Zhang Y, Nielsen R (2019) Genetic rescue and the maintenance of native ancestry. Conserv Genet 20:59-64. https://doi. org/10.1007/s10592-018-1132-1

Hasselgren M, Angerbjörn A, Eide NE, Erlandsson R, Flagstad Ø, Landa A, Wallén J, Norén K (2018) Genetic rescue in an inbred Arctic fox (Vulpes lagopus) population. Proc R Soc B 285:20172814. https://doi.org/10.1098/rspb.2017.2814

Hedrick PW (1994) Purging inbreeding depression and the probability of extinction: full-sib mating. Heredity 73:363-372. https://doi. org/10.1038/hdy. 1994.183

Hedrick PW (2012) What is the evidence for heterozygote advantage selection? Trends Ecol Evol 27:698-704. https://doi.org/10. 1016/j.tree.2012.08.012

Hedrick PW, Fredrickson R (2010) Genetic rescue guidelines with examples from Mexican wolves and Florida panthers. Conserv Genet 11:615-626. https://doi.org/10.1007/s10592-009-9999-5

Hedrick PW, García-Dorado A (2016) Understanding inbreeding depression, purging, and genetic rescue. Trends Ecol Evol 31:940-952. https://doi.org/10.1016/j.tree.2016.09.005

Hedrick PW, Peterson RO, Vucetich LM, Adams JR, Vucetich JA (2014) Genetic rescue in Isle Royale wolves: genetic analysis and the collapse of the population. Conserv Genet 15:1111-1121. https://doi.org/10.1007/s10592-014-0604-1

Hedrick PW, Hellsten U, Grattapaglia D (2016) Examining the cause of high inbreeding depression: analysis of whole-genome sequence data in 28 selfed progeny of Eucalyptus grandis. New Phytol 209:600-611. https://doi.org/10.1111/nph.13639

Hedrick PW, Kardos M, Peterson RO, Vucetich JA (2017) Genomic variation of inbreeding and ancestry in the remaining two Isle Royale wolves. J Heredity 108(2):120-126

Hedrick PW, Robinson JA, Peterson RO, Vucetich JA (2019) Genetics and extinction and the example of Isle Royale wolves. Anim Conserv 22:302-309. https://doi.org/10.1111/acv.12479

Hufbauer RA, Szúcs M, Kasyon E, Youngberg C, Koontz MJ, Richards C, Tuff T, Melbourne BA (2015) Three types of rescue can avert extinction in a changing environment. Proc Natl Acad Sci USA 112:10557-10562. https://doi.org/10.1073/pnas.1504732112

IUCN (International Union for the Conservation of Nature) (2012) IUCN Red List categories and criteria: version 3.1. 2nd edition. IUCN Species Survival Commission, Gland and Cambridge.

Johnson WE et al (2010) Genetic restoration of the Florida panther. Science 329:1641-1645

Kardos M, Shafer AB (2018) The peril of gene-targeted conservation. Trends Ecol Evol 33:827-839. https://doi.org/10.1126/science. 1192891

Keightley PD, Eyre-Walker A (2007) Joint inference of the distribution of fitness effects of deleterious mutations and population demography based on nucleotide polymorphism frequencies. Genetics 177:2251-2261. https://doi.org/10.1534/genetics.107.080663

Keller MC, Visscher PM, Goddard ME (2011) Quantification of inbreeding due to distant ancestors and its detection using dense single nucleotide polymorphism data. Genetics 189:237-249. https://doi.org/10.1534/genetics.111.130922

Kim BY, Huber CD, Lohmueller KE (2017) Inference of the distribution of selection coefficients for new nonsynonymous mutations using large samples. Genetics 206:345-361. https://doi.org/10. 1534/genetics.116.197145

Koen EL, Bowman J, Wilson PJ (2015) Isolation of peripheral populations of Canada lynx (Lynx canadensis). Can J Zool 93(7):521-530

Kolodny O, McLaren MR, Greenbaum G, Ramakrishnan U, Feldman MW, Petrov D, Taylor RW (2019) Reconsidering the management paradigm of fragmented populations. https://doi.org/10. $1101 / 649129$ 
Kyriazis CC, Wayne RK, Lohmueller KE (2020) Strongly deleterious mutations are a primary determinant of extinction risk due to inbreeding depression. Evol Lett 5:33-47. https://doi.org/10. 1002/evl3.209

López-Cortegano E, Vilas A, Caballero A, García-Dorado A (2016) Estimation of genetic purging under competitive conditions. Evolution 70:1856-1870. https://doi.org/10.1111/evo.12983

Miller SM, Druce DJ, Dalton DL, Harper CK, Kotze A, Packer C, Slotow R, Bloomer P (2020) Genetic rescue of an isolated African lion population. Conserv Genet 21:41-53. https://doi.org/10.1007/ s10592-019-01231-y

Mills LS, Allendorf FW (1996) The one-migrant-per-generation rule in conservation and management. Conserv Biol 10:1509-1518. https://doi.org/10.1046/j.1523-1739.1996.10061509.x

Morton NE, Crow JF, Muller HJ (1956) An estimate of the mutational damage in man from data on consanguineous marriages. Proc Natl Acad Sci USA 42:855-863. https://doi.org/10.1073/pnas.42.11.855

Mukai T (1988) Genotype-environment interaction in relation to the maintenance of genetic variability in populations of Drosophila melanogaster. In: Weir BS, Eisen EJ, Goodman MM, Namkoong G (eds) Proceedings of the second international conference on quantitative genetics, Chap. 3, pp. 21-31. Sinauer, Sunderland

Norén K, Godoy E, Dalén L, Meijer T, Angerbjörn A (2016) Inbreeding depression in a critically endangered carnivore. Mol Ecol 25:33093318. https://doi.org/10.1111/mec.13674

O'Grady JJ, Brook BW, Reed DH, Ballou JD, Tonkyn DW, Frankham R (2006) Realistic levels of inbreeding depression strongly affect extinction risk in wild populations. Biol Conserv 133:42-51. https://doi.org/10.1016/j.biocon.2006.05.016

Palstra FP, Ruzzante DE (2008) Genetic estimates of contemporary effective population size: what can they tell us about the importance of genetic stochasticity for wild population persistence? Mol Ecol 17:3428-3447. https://doi.org/10.1111/j.1365-294X.2008.03842.x

Pekkala N, Knott KE, Kotiaho JS, Puurtinen M (2012) Inbreeding rate modifies the dynamics of genetic load in small populations. Ecol Evol 2:1791-1804. https://doi.org/10.1002/ece3.293

Ralls K, Ballou JD, Dudash MR, Eldridge MDB, Fenster CB, Lacy RC, Sunnucks P, Frankham R (2018) Call for a paradigm shift in the genetic management of fragmented populations. Conserv Lett 11:e12412. https://doi.org/10.1111/conl.12412

Ralls K, Sunnucks P, Lacy RC, Frankham R (2020) Genetic rescue: a critique of the evidence supports maximizing genetic diversity rather than minimizing the introduction of putatively harmful genetic variation. Biol Conserv 251:108784. https://doi.org/10.1016/j. biocon.2020.108784

Robert A, Couvet D, Sarrazin F (2003) Bottlenecks in large populations: the effect of immigration on population viability. Evol Ecol 17:213-231. https://doi.org/10.1023/A:1025563107092

Robinson JA, Brown C, Kim BY, Lohmueller KE, Wayne RK (2018) Purging of strongly deleterious mutations explains long-term persistence and absence of inbreeding depression in Island foxes. Curr Biol 28:3487-3494. https://doi.org/10.1016/j.cub.2018.08.066

Robinson JA, Räikkönen J, Vucetich LM, Vucetich JA, Peterson RO, Lohmueller KE, Wayne RK (2019) Genomic signatures of extensive inbreeding in Isle Royale wolves, a population on the threshold of extinction. Sci Adv 5:eaau0757. https://doi.org/10.1126/sciadv. aau0757

Robinson ZL, Bell DA, Dhendup T, Luikart G, Whiteley AR, Kardos M (2020) Evaluating the outcomes of genetic rescue attempts. Conserv Biol. https://doi.org/10.1111/cobi.13596

Saccheri IJ, Brakefield PM (2002) Rapid spread of immigrant genomes into inbred populations. Proc R Soc B 269:1073-1078. https://doi. org/10.1098/rspb.2002.1963

Santiago E, Novo I, Pardiñas AF, Saura M, Wang J, Caballero A (2020) Recent demographic history inferred by high-resolution analysis of linkage disequilibrium. Mol Biol Evol 37:3642-3653. https://doi. org/10.1093/molbev/msaa169
Santos M (1997) On the contribution of deleterious alleles to fitness variance in natural populations of Drosophila. Genet Res 70:105-115

Sharp NP, Agrawal AF (2018) An experimental test of the mutationselection balance model for the maintenance of genetic variance in fitness components. Proc R Soc B 285:20181864. https://doi.org/ 10.1098/rspb.2018.1864

Tallmon DA (2017) Get a move on: the value of rescue. Anim Conserv 20:16-17. https://doi.org/10.1111/acv.12335

Teixeira JC, Huber CD (2021) The inflated significance of neutral genetic diversity in conservation genetics. Proc Natl Acad Sci USA 118:e2015096118. https://doi.org/10.1073/pnas.2015096118

Templeton AR, Read B (1984) Factors eliminating inbreeding depression in a captive herd of Speke's gazelle (Gazella spekei). Zoo Biol 3:177-199. https://doi.org/10.1002/zoo.1430030302

Thurman TJ, Barrett RD (2016) The genetic consequences of selection in natural populations. Mol Ecol 25:1429-1448. https://doi.org/ 10.1111/mec.13559

Van der Valk T, de Manuel M, Marques-Bonet T, Guschanski K (2019) Estimates of genetic load in small populations suggest extensive purging of deleterious alleles. https://doi.org/10.1101/696831

Vilà C, Sundqvist AK, Flagstad $\varnothing$, Seddon J, Bjornerfeldt S, Kojola I, Casulli A, Sand H, Wabakken P, Ellegren H (2003) Rescue of a severely bottlenecked wolf (Canis lupus) population by a single immigrant. Proc R Soc B 270:91-97. https://doi.org/10.1098/rspb. 2002.2184

Vucetich JA, Waite TA, Nunney L (1997) Fluctuating population size and the ratio of effective to census population size. Evolution 51:20172021. https://doi.org/10.2307/2411022

Waller DM (2015) Genetic rescue: a safe or risky bet? Mol Ecol 24:25952597. https://doi.org/10.1111/mec.13220

Waller DM (2021) Addressing Darwin's dilemma: can pseudo-overdominance explain persistent inbreeding depression and load? Evolution 75-4:779-793. https://doi.org/10.1111/evo.14189

Wang J, Hill WG, Charlesworth D, Charlesworth B (1999) Dynamics of inbreeding depression due to deleterious mutations in small populations: mutation parameters and inbreeding rate. Genet Res 74:165-178. https://doi.org/10.1017/S0016672399003900

Weeks AR, Heinze D, Perrin L, Stoklosa J, Hoffmann AA, van Rooyen A, Kelly T, Mansergh I (2017) Genetic rescue increases fitness and aids rapid recovery of an endangered marsupial population. Nat Comm 8:1071. https://doi.org/10.1038/s41467-017-01182-3

Weiser EL, Grueber CE, Kennedy ES, Jamieson IG (2016) Unexpected positive and negative effects of continuing inbreeding in one of the world's most inbred wild animals. Evolution 70:154-166. https:// doi.org/10.1111/evo.12840

Whiteley AR, Fitzpatrick SW, Funk WC, Tallmon DA (2015) Genetic rescue to the rescue. Trends Ecol Evol 30:42-49. https://doi.org/ 10.1016/j.tree.2014.10.009

Wilder AP, Navarro AY, King SN, Miller WB, Thomas SM, Steiner CC, Ryder OA, Shier DM (2020) Fitness costs associated with ancestry to isolated populations of an endangered species. Conserv Genet 21:589-601. https://doi.org/10.1007/s10592-020-01272-8

Xue Y, Prado-Martinez J, Sudmant PH, Narasimhan V, Ayub Q, Szpak $M$ et al (2015) Mountain gorilla genomes reveal the impact of long-term population decline and inbreeding (Supl. 1). Science 348:242-245. https://doi.org/10.1126/science.aaa3952

Zajitschek SRK, Zajitschek F, Brooks RC (2009) Demographic costs of inbreeding revealed by sex-specific genetic rescue effects. BMC Evol Biol 9:289. https://doi.org/10.1126/science.aaa3952

Publisher's Note Springer Nature remains neutral with regard to jurisdictional claims in published maps and institutional affiliations. 